\title{
Measurement of leakage neutron spectra with D-T neutrons and evaluated nuclear data
}

\author{
Rui $\mathrm{Han}^{1,2}$, Zhiqiang Chen ${ }^{1, *}$, Guoyu $\operatorname{Tian}^{1}$, Yangbo $\mathrm{Nie}^{2}$, Fudong Shi ${ }^{1}$, Suyalatu Zhang ${ }^{3}$, Xin Zhang ${ }^{1}$, Bingyan Liu ${ }^{1}$, and \\ Hui $\operatorname{Sun}^{1}$ \\ ${ }^{1}$ Institute of Modern Physics, Chinese Academy of Sciences, Lanzhou, 730000, China \\ ${ }^{2}$ China Nuclear Data Center, China Institute of Atomic Energy, Beijing, 102413, China \\ ${ }^{3}$ College of Physics and Electronics Information, Inner Mongolia University for the Nationalities, Tongliao, 028000, China
}

\begin{abstract}
Benchmarking of evaluated neutron nuclear data libraries was performed for $\sim 14.8 \mathrm{MeV}$ neutrons on the several targets, such as gallium, graphite, silicon carbide, uranium and tungsten samples. The experiments were performed at China Institute of Atomic Energy (CIAE). The neutron leakage spectra from the samples were measured at $60^{\circ}$ and $120^{\circ}$ by a TOF technique with a BC501A scintillation detector. The measured spectra are rather well reproduced by MCNP-4C simulations with the CENDL3.1, JENDL-4.0 and the new release ENDF/B-VIII.0, JEFF-3.3 evaluated nuclear data libraries and so on. There have some difference between experiments and simulations for the elastic and inelastic contributions in the partial energy range. And the discrepancies of the neutron leakage spectra in the MCNP simulations originate simply from the differences in the spectra distributions of the neutron reaction channels in the evaluated nuclear data libraries.
\end{abstract}

\section{Introduction}

The experimental studies of fast neutron scattering are important for design of nuclear reactors $[1,2]$ and accelerator driven subcritical systems (ADS) [3, 4]. They play a crucial role for verification of the evaluated nuclear data libraries, especially some elements that are of interest in ADS, fission and fusion reactor technologies, such as spallation target material (gallium and tungsten), structural material (graphite and silicon carbide), fission fuel (uranium) and so on. For these nuclear engineering design, not only precise and reliable nuclear data, but also detailed study of the neutronics are required. However, some discrepancies between measured neutron leakage spectra and MCNP calculated ones have been observed for some target samples [1]. Their available experimental and calculation data are limited because of few benchmark experiments. So the neutronics study and benchmarking of the evaluated nuclear data libraries are necessary. The benchmarking experiment has been performed at China Institute of Atomic Energy (CIAE) since 2009 [5]. The validity of the benchmarking test system has been examined. Some targets have been studied, such as U [5], Be [6], polyethlene [7] and so on. These research work are important for the improvement of nuclear data, benchmarking of neutron evaluated nuclear data and validation of nuclear reaction models. It has also very important application in the design of ADS.

In this paper, we presented some results of integral neutronics experiments that were investigated at Institute

*e-mail: zqchen@impcas.ac.cn of Modern Physics, CAS in order to validate evaluate nuclear data related to the materials used in ADS.

\section{Measurements and simulations}

A series of integral neutronics experiments were performed at CIAE. The neutron leakage spectra were measured at $60^{\circ}$ and $120^{\circ}$ by a TOF technique with a BC501A scintillation detector. A schematic view of the experimental arrangement is shown in the figure 1. The details of the experimental setup and the data acquisition system can be found in Refs [5, 8, 9]. Benchmarking of the evaluated neutron nuclear data libraries was conducted on ADS relevant materials gallium, graphite, silicon carbine, tungsten, uranium samples and so on. The neutron leakage spectra are simulated by MCNP-4C [10] code. The recent release ENDF/B-VIII.0 and JEFF-3.3 evaluate nuclear data libraries, and CENDL-3.1, JENDL- 4.0 libraries were validated. In the following section, the comparisons between MCNP code simulated results and the experimental data from target samples are presented.

\section{Results and discussion}

\subsection{Gallium}

Gallium (Ga) sample was made as a cylindrical shape with $\Phi 13 \mathrm{~cm}^{2} \times 6.4 \mathrm{~cm}$. And natural Ga was used, which is composed of $60.11 \%{ }^{69} \mathrm{Ga}$ and $39.89 \%{ }^{71} \mathrm{Ga}$. The neutron leakage spectra from Ga sample at $60^{\circ}$ and $120^{\circ}$ are shown in the figure 3 and figure 4, respectively. In the figure, dots represent the experimental results, whereas different 


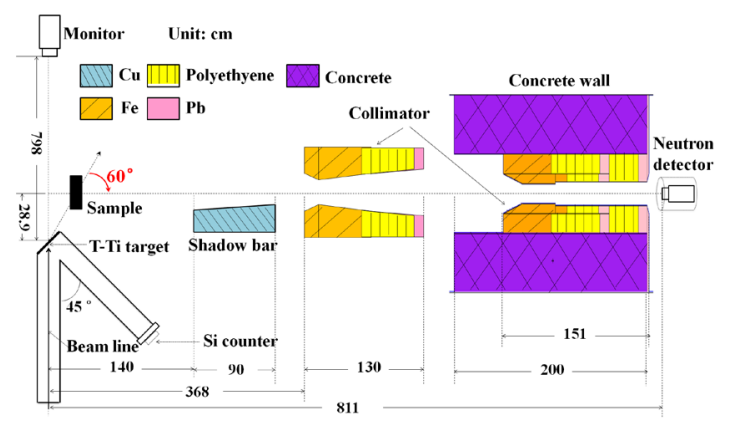

Figure 1. A schematic view of the experimental arrangement.

lines are those from the MCNP simulations using the evaluated databases of ENDF/B-VIII.0 (long-dashed), JEFF3.3 (dashed), CENDL3.1 (dashed-dot) and JENDL4.0 (dashed-dot-dot). The errors presented in the figure are from the statistical and systematic errors. The new major release VIII.0 of the ENDF/B and JEFF-3.3 nuclear data libraries have been tested using benchmark calculations.

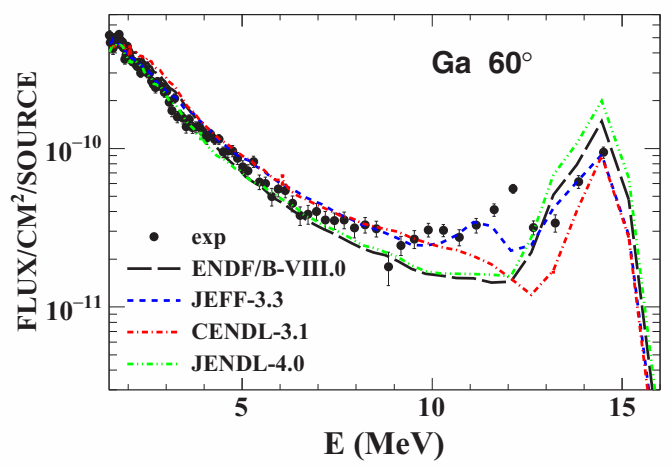

Figure 2. Neutron leakage spectra for Ga sample at $60^{\circ}$.

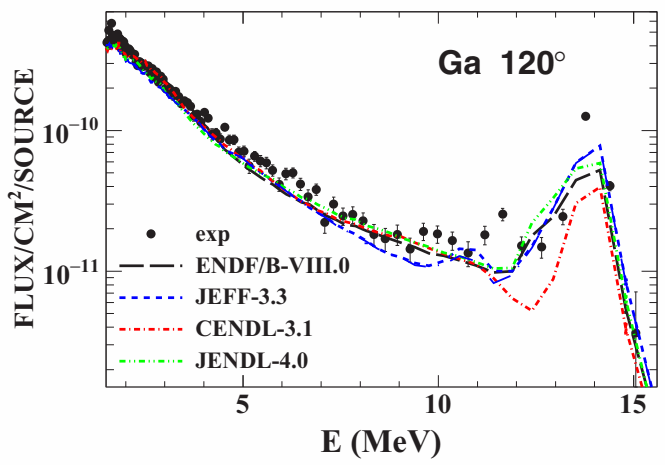

Figure 3. Neutron leakage spectra for Ga sample at $120^{\circ}$.

From the figure 2 and figure 3, one can see that the experimental neutron leakage spectra are well reproduced by the four evaluate nuclear data libraries, except for the inelastic contributions at $\mathrm{E}_{n} \sim 12 \mathrm{MeV}$. For the inelastic scattering peak around $12 \mathrm{MeV}$, which are dominated by the discrete levels, JEFF-3.3 library shows a slightly better agreement with the measured data. For Ga benchmark, the results of ENDF/B-VIII.0 library are same with ENDF/BVII.1 library, see the Ref. [8]. And JEFF-3.3 library have improved around $10 \mathrm{MeV}$ comparing before release version, see the Ref. [11].

\subsection{Graphite}

Graphite (C) sample was made as a cylindrical shape with $\Phi 13 \mathrm{~cm}^{2} \times 20 \mathrm{~cm}$ with average weight density of 1.833 $\mathrm{g} / \mathrm{cm}^{3}$. The figure 4 and figure 5 show that the neutron leakage spectra from $\mathrm{C}$ sample at $60^{\circ}$ and $120^{\circ}$, respectively. The results show that the experimental neutron leakage spectra are well reproduced by the four evaluated nuclear data libraries, except below $3 \mathrm{MeV}$ enrgy range with the ENDF/B-VIII.0 and JEFF-3.3 libraries. The results of other evaluated nuclear data libraries see the Ref. [12, 13].

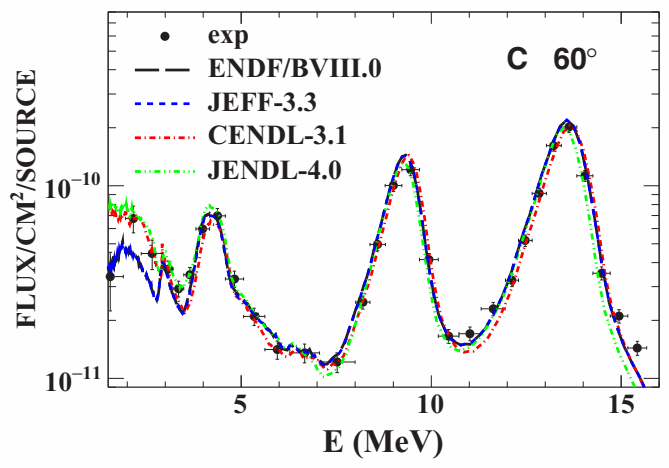

Figure 4. Neutron leakage spectra for $\mathrm{C}$ sample at $60^{\circ}$.

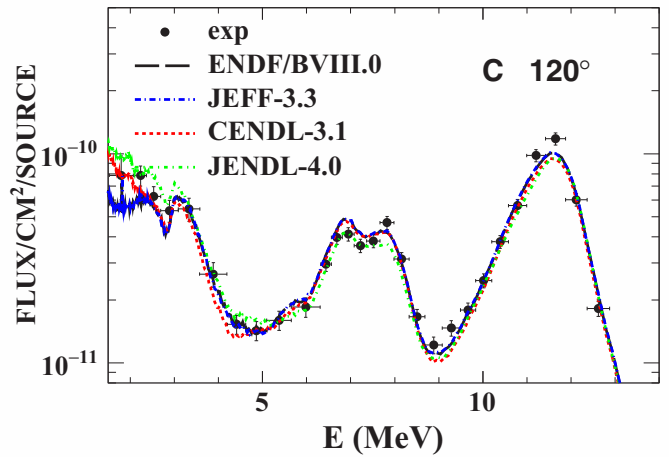

Figure 5. Neutron leakage spectra for $\mathrm{C}$ sample at $120^{\circ}$.

\subsection{Silicon carbide}

Silicon carbide ( $\mathrm{SiC}$ ) sample was made as a cylindrical shape with $\Phi 13 \mathrm{~cm}^{2} \times 20 \mathrm{~cm}$ with average weight density of $1.722 \mathrm{~g} / \mathrm{cm}^{3}$. The figure 6 and figure 7 show that the neutron leakage spectra from $\mathrm{SiC}$ sample at $60^{\circ}$ and $120^{\circ}$, respectively. The results show that the experimental neutron leakage spectra are well reproduced by 
the four evaluated nuclear data libraries in the whole energy range. The ENDF/B-VIII.0 library results show better agreements with the experimental data than those calculated with libraries else. More details results see the Ref. $[14,15]$.

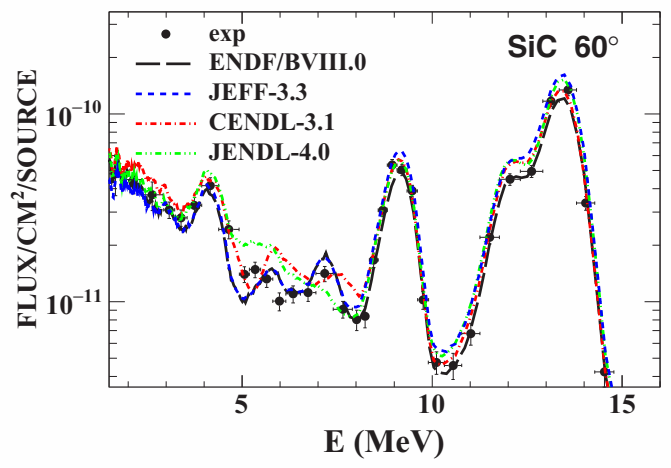

Figure 6. Neutron leakage spectra for $\mathrm{SiC}$ sample at $60^{\circ}$.

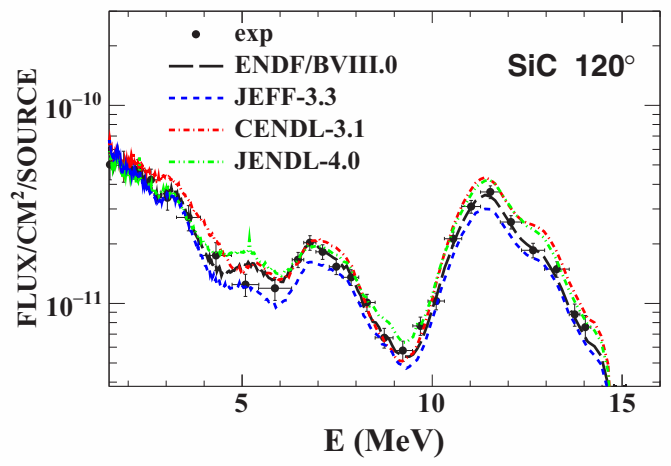

Figure 7. Neutron leakage spectra for $\mathrm{SiC}$ sample at $120^{\circ}$.

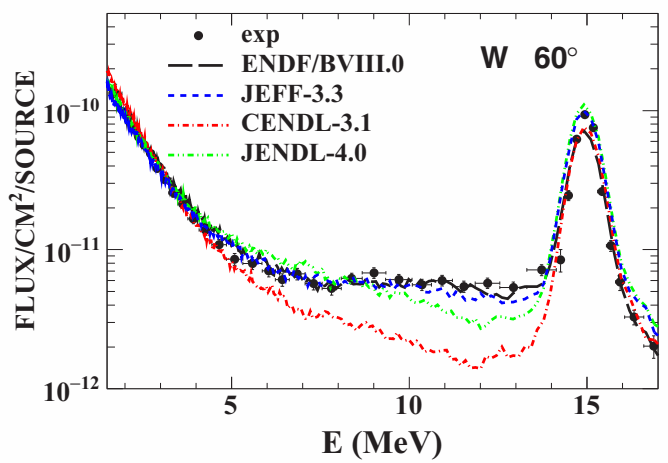

Figure 8. Neutron leakage spectra for W sample at $60^{\circ}$.

\subsection{Tungsten}

The neutron leakage spectra for a slab tungsten (W) sample with the size of $10 \times 10 \times 7 \mathrm{~cm}^{3}$ at $60^{\circ}$ and $120^{\circ}$ are shown in the figure 8 and figure 9 , respectively. The results show that the experimental neutron leakage spectra are

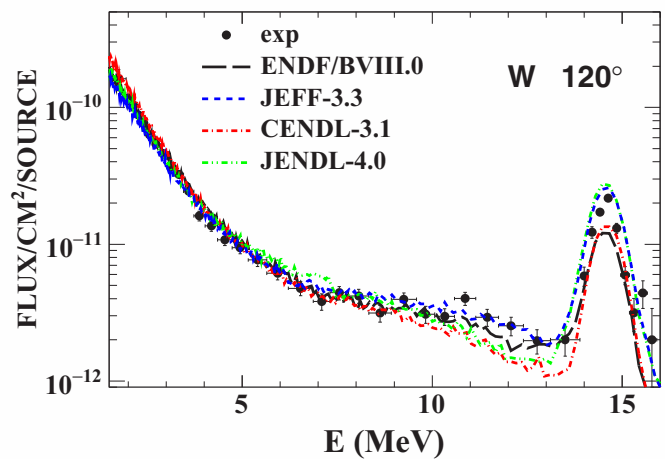

Figure 9. Neutron leakage spectra for W sample at $120^{\circ}$.

well reproduced by ENDF/B-VIII.0 and JEFF-3.3 evaluated nuclear data libraries in the whole energy range. The results from ENDF/B-VIII.0 library slightly underestimate the cross section in elastic scattering peak. And the results from CENDL-3.1 library significantly underestimate the cross section in about 8-13 MeV energy range. More details results and the other evaluated nuclear data libraries results see the Ref. [16, 17].

\subsection{Uranium}

The neutron leakage spectra for a slab uranium (U) sample with the size of $10 \times 10 \times 11 \mathrm{~cm}^{3}$ at $60^{\circ}$ and $120^{\circ}$ are shown in the figure 10 and figure 11 , respectively. The results show that the experimental neutron leakage spectra can be reproduced, and the simulations with the JENDL4.0 libraries show better agreements with the experimental ones than other evaluated nuclear data libraries. The results from CENDL-3.1 library overestimate the cross section in 5-8 MeV energy range and around $13 \mathrm{MeV}$. And the ENDF/B-VIII.0 and JEFF-3.3 libraries simulated results are similar. The other evaluated nuclear data libraries results see the Ref. [18].

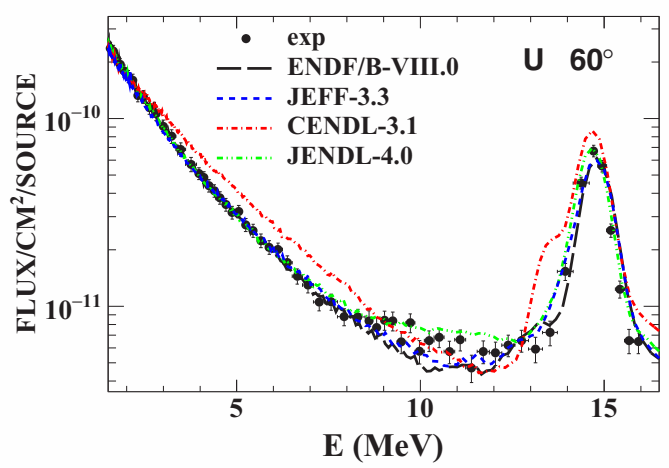

Figure 10. Neutron leakage spectra for U sample at $60^{\circ}$.

\section{Summary}

The neutron leakage spectra from several samples $(\mathrm{Ga}, \mathrm{C}$, $\mathrm{SiC}, \mathrm{W}$ and $\mathrm{U}$ ) relevant for ADS were measured by a TOF 


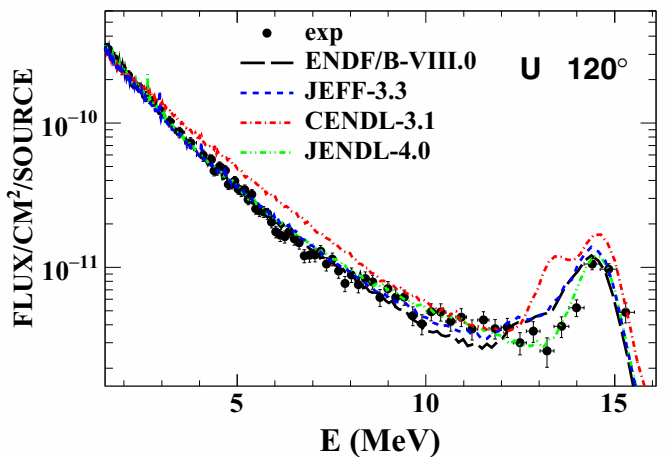

Figure 11. Neutron leakage spectra for U sample at $120^{\circ}$.

technique at $60^{\circ}$ and $120^{\circ}$. Benchmarking of evaluated nuclear data libraries on target samples were investigated using MCNP-4C simulations with CENDL-3.1, JENDL-4.0 libraries and the new release ENDF/B-VIII.0 and JEFF-3.3 libraries. The results show that the essential characteristic properties of the experimental spectra are well reproduced by the these simulations. But the difference between simulated ones can be observed in the partial energy range. For example, the significant discrepancies are observed around $12 \mathrm{MeV}$ energy range for $\mathrm{Ga}$ and $\mathrm{W}$ samples, and below $3 \mathrm{MeV}$ energy range for $\mathrm{C}$ sample. The largely overestimate of simulations with CENDL-3.1 library are observed in 5-8 MeV energy range and around $13 \mathrm{MeV}$. These discrepancies of the neutron leakage spectra in the MCNP simulations originate simply from the differences in the spectra distributions of the neutron reaction channels in the evaluated nuclear data libraries.

\section{References}

[1] C Steven, van der Marck, Nucl. Data Sheets 113, 2935 (2012)
[2] Hiroyuki Hashikura, et al., J. Nucl. Sci. Technol 23(6), 477 (1986)

[3] A J Koning, J -P Delaroche, O Bersillon. Nucl. Instrum. Methods Phys. Res. A 41449 (1998)

[4] L Yang, W L Zhan, Sci. China Technol. Sci 58, 1705 (2015)

[5] Y Nie, J Bao, X Ruan, et al., Annals of Nuclear Energy 37, 1456 (2010)

[6] Y Nie et al., Annual Report of China Institute of Atomic Energy 78 (2013)

[7] Y Nie et al., Nuclear Physics Review 29(3), 310 (2012)

[8] R Han, R Wada, Z Chen, et al., Nuclear Physics A 936, 17 (2015)

[9] R Han, Z Chen, Y Nie, et al., Fusion Engineering and Design 135, 116 (2018)

[10] J Briesmeister, MCNP-A General Monte Carlo Nparticle Transport Code, Version 4C (Tech. Rep. LA, M. Los Alamos National Laboratory, USA, 2000) 13709

[11] R Han, Z Chen, Y Nie, et al., Atomic Energy Science and Technology 52, 1200 (2018)

[12] F Luo, R Han, Z Chen, et al., Applied Radiation and Isotopes 116, 185 (2016)

[13] F Luo, R Han, Z Chen, et al., Nuclear Engineering and Design 331, 342 (2018)

[14] F Luo, R Han, Y Nie, et al., Fusion Engineering and Design 112, 355 (2016)

[15] F Luo, R Han, Z Chen, et al., Nuclear Engineering and Design 331, 342 (2018)

[16] S Zhang, Z Chen, Y Nie, et al., Fusion Eng.Des 92, $41(2015)$

[17] S Zhang, Y Nie, J Ren, et al., NUCL SCI TECH 28 , 27 (2017)

[18] Q Sun, Z Chen, R Han, et al., Fusion Engineering and Design 125, 9 (2017) 